\title{
Capacidade funcional em idosos com doença de parkinson: revisão de literatura
}

\author{
Functional capacity in the elderly with parkinson's disease: \\ literature review
}

RESUmo A doença de Parkinson (DP) consiste em uma enfermidade crônica e progressiva, caracterizada pela depleção da dopamina por degeneração dos neurônios da substância negra, que leva a perdas motoras e cognitivas. Pacientes com DP apresentam intolerância ao esforço físico com consequente comprometimento de sua capacidade funcional (CF). O objetivo deste estudo foi analisar a eficácia do teste de caminhada de seis minutos (TC6) como ferramenta para a avaliação da CF de idosos com DP. Foi realizada uma revisão de literatura nas bases de dados PubMed, SciELO e LILACS, com artigos publicados no período de janeiro de 2005 a dezembro de 2015, por meio do cruzamento dos descritores physical therapy, exercise test e o termo functional capacity com o descritor Parkinson's disease. Foram identificados cinco estudos que avaliaram 203 idosos, dos quais 166 acometidos pela DP. Entre os idosos com DP, a idade média foi de $66,6 \pm 2,8$ anos e a distância média percorrida no TC6 variou entre 332 a 546 metros. Os estudos concluíram que os idosos com DP apresentaram redução da CF. A análise feita nesta revisão sugere que o teste de caminhada de seis minutos não parece a ferramenta mais eficaz para a avaliação da capacidade funcional de idosos com doença de Parkinson, pois pode subestimar a sua funcionalidade.

Palavras-chave: Idoso. Doença de Parkinson. Teste de Esforço. FiSIOTERAPIA.

Abstract Parkinson's disease (PD) consists of a chronic and progressive disorder characterized by depletion of dopamine due to degeneration of nigral neurons leading to cognitive and motor losses. Patients with PD present an intolerance to physical effort, leading to impaired functional capacity (FC). The aim of this study was to analyze the efficacy of the six-minute walk test (6MWT) as a tool for assessing CF in the elderly with PD. A literature review was performed in the PubMed, SciELO and LILACS databases, from January 2005 to December 2015, through the cross-referencing of the physical therapy, exercise test and functional capacity descriptors with the descriptor Parkinson's disease. Five studies evaluating 203 elderly were identified, of which 166 were affected by PD. Among the elderly with PD, the mean age was $66.6 \pm 2.8$ years and the mean distance covered in the 6MWT ranged from 332 to 546 meters. The studies concluded that the elderly with $\mathrm{PD}$ presented reduction of CF. The analysis performed in this review suggests that the six-minute walk test seems not to be the most effective tool for assessing the functional capacity of elderly people with Parkinson's disease, as it may underestimate its functionality.

Odete Mauad Cavenaghi ${ }^{1}$

Key-words: Elderly. Parkinson's disease. Exercise Test. PhysiotheRAPY. 


\section{INTRODUÇÃO}

A doença de Parkinson (DP) consiste em uma enfermidade crônica e progressiva, que acomete, em geral, a população idosa na faixa etária entre 50 a 70 anos. ${ }^{1,2} \mathrm{~A}$ DP caracteriza-se pela depleção da dopamina por degeneração dos neurônios da substância negra, o que gera perdas motoras e cognitivas. Tremor de repouso, rigidez, bradicinesia ou hipocinesia e instabilidade postural são os principais acometimentos encontrados em pacientes com DP. ${ }^{3,4}$

A DP é mais comum em indivíduos do sexo masculino, sem diferenças entre grupos étnicos. ${ }^{5,6} \mathrm{~A}$ prevalência mundial da doença gira em torno de $1 \%$ a $2 \% .^{5}$ No Brasil, o número de indivíduos com DP é estimado em 200 mil casos por ano, com prevalência semelhante à verificada no mundo. ${ }^{7}$

Com o envelhecimento fisiológico, ocorre uma diminuição gradativa de massa corporal, função pulmonar, força e endurance muscular respiratória e periférica, e da capacidade física, o que explica os efeitos debilitantes. ${ }^{8}$ Quando associados à DP, observa-se uma crescente perda da funcionalidade, que afeta ainda mais o estado nutricional com piora da qualidade de vida (QV) e da independência funcional do indivíduo. ${ }^{9}$

Pacientes com DP apresentam redução significativa das fibras musculares do tipo IIA, o que gera à atrofia por desuso ou descondicionamento físico e predispõe à fraqueza e fadiga musculares. ${ }^{10} \mathrm{~A}$ redução de massa muscular leva à incoordenação dos movimentos, quedas frequentes, intolerância ao exercício e piora progressiva do condicionamento físico, fato que limita as atividades de vida diária (AVD). ${ }^{10}$

Uma vez que esses pacientes apresentam intolerância ao esforço físico, com comprometimento na execução de AVD simples, como a caminhada, faz-se necessário o emprego de testes específicos para a avaliação da capacidade funcional (CF) nessa população. O teste de caminhada de seis minutos (TC6) é considerado uma das formas alternativas de avaliação da CF, por ser de fácil realização e baixa complexidade, além de não requerer equipamentos de alto custo na prática clínica. ${ }^{11-13}$

Contudo, são escassos os estudos que analisaram as variáveis que influenciam a capacidade física de pacientes com DP, avaliada pelo TC6. Além disso, buscas nas bases de dados pertinentes não identificaram estudos de revisão que sistematizassem a utilização do TC6 em pacientes com DP. A aplicação desse teste compreende uma das etapas de uma avaliação física minuciosa imprescindível para estabelecer um programa de tratamento fisioterapêutico adequado às limitações e à gravidade da doença.

Dessa forma, o objetivo do presente estudo foi analisar a eficácia do teste de caminhada de seis minutos como ferramenta para a avaliação da capacidade funcional de idosos com doença de Parkinson.

\section{MÉTODOS}

Este estudo caracteriza-se como uma revisão de literatura. ${ }^{14}$

\section{Estratégia de busca}

Esta revisão de literatura utilizou artigos selecionados em outubro de 2016, a partir de consultas às bases de dados PubMed, SciELO e Lilacs, por artigos publicados no período de janeiro de 2005 a dezembro de 2015. Para a busca foram utilizadas as palavras-chave: fisioterapia, avaliação geriátrica e teste de esforço em cruzamento, por meio do operador booleano "and", com o descritor doença de Parkinson, as quais fo- 
ram definidas com base nos Descritores em Ciências da Saúde (DeCS) para pesquisa nas bases SciELO e Lilacs e os seus correspondentes na língua inglesa, Medical Subject Headings (MeSH) physical therapy, exercise test e o termo functional capacity com o descritor Parkinson's disease para pesquisa na base PubMed.

O termo capacidade funcional, não considerado um descritor pelo DeCS, foi incluso devido à sua larga utilização como palavra-chave. Todas as referências dos estudos selecionados foram também revisadas para complementação da pesquisa. Todas as etapas da busca foram realizadas por somente um avaliador.

\section{Critérios de inclusão e exclusão}

Foram incluídos estudos publicados nos últimos dez anos, nas línguas inglesa e portuguesa, com seres humanos acima de 60 anos de idade, portadores de DP, em qualquer estágio da doença, que foram submetidos à avaliação da CF por meio do TC6. Foram inclusos ensaios clínicos randomizados e não randomizados, estudos longitudinais e estudos transversais.

Resumos de dissertações ou teses acadêmicas, estudos com crianças, adolescentes ou adultos, trabalhos sem disponibilidade de texto completo livre em algum sítio da internet e estudos que utilizaram outros testes para avaliar a capacidade física, foram excluídos.

\section{Estratégia de seleção}

Para a seleção dos artigos, inicialmente foi feita a triagem dos títulos relacionados ao tema em questão. Essa seleção foi baseada nos títulos que abordassem como ideia principal: a aplicação do TC6 em idosos com DP. Ao final da busca, foram excluídos os títulos repetidos, já que esta foi realizada em diversas bases de dados. Em seguida, foi feita a leitura detalhada dos resumos dos artigos, a fim de selecionar aqueles que abordassem exclusivamente a utilização do TC6 para avaliar a CF na DP. Excluídos os resumos que não versavam sobre o tema, os textos completos foram analisados e os que não se enquadravam nos critérios de exclusão foram inclusos como resultado final da busca.

\section{AnÁlise dos Dados}

Os dados foram analisados de forma qualitativa e apresentados na forma de tabela com a descrição das seguintes características: autor do estudo, objetivo do estudo, características clínicas da população, distância percorrida no TC6 e conclusões encontradas.

\section{RESUlTADOS}

A busca nas bases de dados, por meio dos descritores selecionados, resultou em 253 títulos, 46 na base SciELO, 56 na Lilacs e 151 na PubMed. A primeira seleção eliminou 213 títulos. Em seguida, procedeu-se à análise do conteúdo dos resumos dos 40 estudos restantes, dos quais foram eliminados 35 por não atenderem aos critérios previamente estabelecidos. Os cinco artigos restantes foram lidos na íntegra e compuseram esta revisão. ${ }^{15-19}$

$\mathrm{Na}$ Tabela 1, encontram-se sumarizados os artigos identificados nesta revisão. Ao analisar as variáveis dos cinco artigos encontrados, verifica-se que foram avaliados 203 idosos, dos quais 166 acometidos pela DP e 37 idosos saudáveis. A idade média dos idosos com DP entre os estudos foi de 67,05 anos e a faixa de distância percorrida no TC6 entre os idosos com DP foi de 332 a 546 metros (m). Todos os estudos concluíram que os idosos com DP apresentaram redução da capacidade física. 
Tabela 1: Características dos estudos que utilizaram o teste de caminhada de seis minutos em idosos com doença de Parkinson.

\begin{tabular}{|c|c|c|c|c|}
\hline Autor & Objetivo & $\begin{array}{l}\text { Características } \\
\text { população }\end{array}$ & $\begin{array}{l}\text { Distância no } \\
\text { TC6 }\end{array}$ & Conclusões \\
\hline Silva et al. ${ }^{15}$ & $\begin{array}{l}\text { Avaliar a funcionalidade, } \\
\text { incapacidade e QV dos } \\
\text { pacientes com DP em } \\
\text { atendimento fisioterapêutico } \\
\text { em um hospital universitário } \\
\text { no Rio de Janeiro }\end{array}$ & $\begin{array}{l}29 \text { idosos, } 62,7 \% \text { homens, } \\
\text { idade média } 67 \pm 7,59 \\
\text { anos }\end{array}$ & $335 \pm 39 \mathrm{~m}$ & $\begin{array}{l}\text { Os idosos avaliados apresentaram } \\
\text { risco de queda diminuído, bom } \\
\text { estado cognitivo e emocional, QV } \\
\text { moderada e pouca dificuldade para } \\
\text { a marcha e realização de AVD }\end{array}$ \\
\hline $\begin{array}{l}\text { Bonjorni et } \\
\text { al. }{ }^{16}\end{array}$ & $\begin{array}{l}\text { Avaliar e comparar o IMMC, } \\
\text { variáveis espirométricas, } \\
\text { força muscular respiratória } \\
\text { e capacidade física, em } \\
\text { pacientes com DP e idosos } \\
\text { saudáveis. Além disso, } \\
\text { verificar quais dessas variáveis } \\
\text { influenciam a capacidade } \\
\text { física de pacientes com DP }\end{array}$ & $\begin{array}{l}25 \text { idosos, de ambos os } \\
\text { sexos, sendo } 10 \text { pacientes } \\
\text { no GDP }-72,7 \pm 10 \text { anos; } \\
\text { e } 15 \text { no GS }-64,8 \pm 6,7 \\
\text { anos }\end{array}$ & $\begin{array}{l}\text { GDP } \\
332,1 \pm 135,5 \\
\mathrm{~m} \\
\text { GS } \\
602,3 \pm 85,3 \mathrm{~m}\end{array}$ & $\begin{array}{l}\text { O GDP apresentou redução da } \\
\text { massa muscular, da endurance e } \\
\text { forças musculares respiratórias, } \\
\text { e da capacidade física, se } \\
\text { comparados aos idosos saudáveis. } \\
\text { Além disso, a PEmáx e a VVM } \\
\text { influenciam a capacidade física } \\
\text { medida pelo TC6, assim como } \\
\text { a massa muscular influencia } \\
\text { a endurance da musculatura } \\
\text { respiratória nos pacientes com DP }\end{array}$ \\
\hline $\begin{array}{l}\text { Scalzo et } \\
\text { al. }{ }^{17}\end{array}$ & $\begin{array}{l}\text { Avaliar o impacto das } \\
\text { mudanças no equilíbrio e na } \\
\text { capacidade de marcha em } \\
\text { doentes com DP }\end{array}$ & $\begin{array}{l}36 \text { idosos, ambos os sexos, } \\
\text { com duração média da } \\
\text { doença de } 7,3 \text { anos, idade } \\
\text { média de } 65,5 \pm 7,9 \text { anos }\end{array}$ & $\begin{array}{l}396,3 \pm 109,9 \\
m\end{array}$ & $\begin{array}{l}\text { O prejuízo no equilíbrio durante a } \\
\text { realização de atividades funcionais } \\
\text { e a redução na capacidade de } \\
\text { caminhar são fatores importantes } \\
\text { que afetam negativamente a } \\
\text { percepção da QV em pacientes } \\
\text { com DP }\end{array}$ \\
\hline $\begin{array}{l}\text { Falvo et } \\
\text { al. }{ }^{18}\end{array}$ & $\begin{array}{l}\text { Determinar a distância no } \\
\text { TC6 em indivíduos com DP e } \\
\text { determinar as deficiências que } \\
\text { contribuem para a capacidade } \\
\text { de caminhar }\end{array}$ & $\begin{array}{l}75 \text { idosos, } 71 \% \text { homens, } \\
\text { com idade média de } 65,09 \\
\pm 9,5 \text { anos }\end{array}$ & $391,6 \pm 99,9 \mathrm{~m}$ & $\begin{array}{l}\text { A distância no TC6 em indivíduos } \\
\text { com DP é explicada em parte } \\
\text { pelas características específicas da } \\
\text { doença e, talvez, em maior medida } \\
\text { pelo equilíbrio prejudicado e } \\
\text { predisposição à queda }\end{array}$ \\
\hline $\begin{array}{l}\text { Canning et } \\
\text { al. }{ }^{19}\end{array}$ & $\begin{array}{l}\text { Examinar a capacidade } \\
\text { de caminhada em pessoas } \\
\text { com DP leve a moderada, } \\
\text { para determinar se as } \\
\text { anormalidades espaço- } \\
\text { temporais observadas } \\
\text { quando as pessoas com DP } \\
\text { percorrem distâncias curtas } \\
\text { são exacerbadas em distâncias } \\
\text { maiores e se essas e outras } \\
\text { alterações motoras afetam a } \\
\text { capacidade de caminhar }\end{array}$ & $\begin{array}{l}38 \text { idosos, GDP }(\mathrm{n}=16) \\
\text { com idade média de } 65 \\
\text { anos e GS }(\mathrm{n}=22) \text { com } \\
\text { idade média de } 66 \text { anos }\end{array}$ & $\begin{array}{l}\text { GDP } \\
546 \pm 103 \mathrm{~m} \\
\text { GS } \\
619 \pm 69 \mathrm{~m}\end{array}$ & $\begin{array}{l}\text { Mesmo quando os idosos com } \\
\text { DP são capazes de caminhar a } \\
\text { velocidades comparáveis aos } \\
\text { controles saudáveis, eles não } \\
\text { sustentam essa velocidade em } \\
\text { distâncias maiores }\end{array}$ \\
\hline
\end{tabular}

Abreviaturas: TC6 = teste de caminhada de seis minutos; DP = doença de Parkinson; $\mathrm{QV}=$ qualidade de vida; $\mathrm{m}=$ metros; $\mathrm{AVD}=$ atividade de vida diária; $\mathrm{IMMC}$ = índice de massa magra corporal; GDP = grupo doença de Parkinson; GS = grupo saudável; PEmáx = pressão expiratória máxima; VVM = ventilação voluntária máxima. 


\section{DIscussão}

Este estudo analisou a eficácia do TC6 como método para avaliar a CF de idosos com DP. Foram identificados apenas cinco estudos na literatura que utilizaram o TC6 como ferramenta de avaliação. Os estudos demonstraram que idosos parkinsonianos apresentaram decréscimo na CF. Contudo, os estudos analisados não avaliaram exclusivamente a CF desses idosos.

Estudo recente avaliou a funcionalidade por meio do TC6, incapacidade e a QV de pacientes idosos com DP em atendimento fisioterapêutico hospitalar. A distância média caminhada pelos idosos foi de $335 \pm$ $39 \mathrm{~m}$. Os autores não relataram o quanto esses valores representaram em percentual do predito para esses idosos. Contudo, os autores disseram que os idosos avaliados apresentaram independência na execução de suas AVD. ${ }^{15} \mathrm{O}$ estudo de Silva et al. ${ }^{15}$ verificou desfechos importantes em idosos com DP, os autores utilizaram além do TC6 para avaliar a CF, a escala unificada de avaliação para a DP (UPDRS) que determina o estadiamento da doença e o nível de dependência para as AVDs. Contudo, importante limitação no estudo reside na ausência de análise de associação ou correlação entre esses dois instrumentos de avaliação.

Bonjorni et al. ${ }^{16}$ compararam, entre outros fatores, a CF por meio do TC6 em pacientes com DP e idosos saudáveis, e observaram que os idosos com DP percorreram distância significativamente menor que os idosos saudáveis, com redução de aproximadamente $270 \mathrm{~m}$, sendo considerado um fator clinicamente importante e significativo. Os autores atribuíram essa diferença à fraqueza muscular periférica presente nos pacientes com DP. Entretanto, outro fator importante que não foi levado em consideração neste estudo é o estágio da doença, pois evidências da literatura apontam que pacientes com DP de estágio leve a moderado mostram melhor prognóstico para a capacidade de exercício normal com exercício aeróbio regular. ${ }^{18}$ Desse modo, pode-se inferir que idosos com DP em estágio leve e moderado apresentariam maior distância caminhada no TC6 que seus pares em estágio grave.

Ainda em relação ao estudo citado, foi verificado que a força muscular expiratória e a ventilação voluntária máxima (VVM) influenciaram a CF medida pelo TC6 nesses idosos. ${ }^{16}$ Segundo os autores, quanto maior a força e a endurance musculares expiratórias, tanto maior a $\mathrm{CF}$, tal relação pode ser explicada pela redução significativa das fibras musculares do tipo IIA presentes nos pacientes com DP, que predispõem à fraqueza muscular periférica e respiratória, e a diminuição da VVM, associada à bradicinesia e rigidez da caixa torácica, bem como, à redução de massa muscular, que gera incoordenação dos movimentos e quedas frequentes, resultante em intolerância ao exercício nesses pacientes. A VVM na DP é considerada importante, pois demonstra a dificuldade de coordenação muscular para a realização de movimento rápido e repetitivo, ${ }^{20}$ além de ser um indicador de doença neuromuscular. ${ }^{21}$

Outras evidências ${ }^{17}$ encontradas nesta revisão apontaram que idosos portadores de DP apresentaram menores distâncias percorridas no TC6, o que se correlacionou com pior percepção da QV. Foram avalia- 
dos idosos com duração média da doença de 7,3 anos e a distância média foi de 396 $\mathrm{m}$ no TC6, que corresponde a apenas $42 \%$ da distância predita, com base em valores normativos para idade e sexo. Segundo os autores, esses achados podem ser explicados pela hipocinesia, que acarreta descondicionamento, limitação e inabilidades físicas graduais. ${ }^{17}$ Houve associação entre menor distância caminhada e pior percepção de QV nos idosos com DP, tal associação pode ter ocorrido em detrimento da percepção dos pacientes sobre sua real mobilidade. Os achados deste estudo demonstram o impacto da DP na CF de seus portadores, que pode limitar não só as AVDs como também as atividades laborais e resultar até em problemas conjugais com maior estigmatização.

Falvo et al. ${ }^{18}$ foram os únicos autores que objetivaram além de determinar a distância percorrida no TC6 em idosos parkinsonianos, identificar os fatores que contribuem para a capacidade física funcional nessa população. A distância média caminhada nessa amostra foi $392 \mathrm{~m}$, o equilíbrio e a mobilidade prejudicados aumentaram a predisposição a quedas e contribuíram para os déficits funcionais observados. Esses achados encontram-se abaixo do valor predito, segundo equações de normalidade. Os fatores predeterminantes para essa perda de CF podem ser explicados pela gravidade da doença, pelo nível de atividade física e pelo equilíbrio em idosos parkinsonianos. ${ }^{18} \mathrm{~A}$ literatura aponta que idosos com DP apresentam distância entre 315 a 560 m no TC6 $6^{19,22-24}$ e alguns fatores podem explicar essa variabilidade, como a gravidade da doença e o nível de atividade física basal. O índice de variância pode chegar a $55 \%$ e pode ser explicado por variáveis independentes combinadas.

Outros autores $^{19}$ analisaram a distância caminhada por idosos com DP leve e moderada, para avaliar se as anormalidades identificadas quando percorridas distâncias menores são exacerbadas em distâncias maiores e se essas e outras alterações motoras afetam a CF. Os idosos com DP caminharam 546 m em média, distância estatisticamente menor que o grupo controle. No grupo com DP, $94 \%$ da variância na capacidade de caminhar foi explicada pela hipocinesia durante a marcha e nas rotações, bem como, aos déficits de força. Os resultados deste estudo demonstram a importância de avaliar a CF nessa população com base no estadiamento da doença, e deixam evidente que à medida que a doença evolui e surgem as alterações inerentes à mesma, maior o comprometimento funcional.

A distância média percorrida no TC6 entre os idosos com DP variou entre 332 $\mathrm{m}$ a $546 \mathrm{~m}$ entre os cinco estudos incluídos nesta revisão. Essa distância corresponde a aproximadamente $45 \%$ da distância prevista com base em valores normativos para idade, sexo, altura e peso. ${ }^{16} \mathrm{O}$ que caracteriza importante comprometimento funcional nessa população.

A CF avalia o potencial funcional do indivíduo e verifica o grau de autonomia para a realização das AVD que, no caso do idoso, encontra-se limitada devido ao processo fisiológico do envelhecimento, fator que pode ser potencializado pela presença de doenças crônicas como a DP. ${ }^{25}$ Dessa forma, pode-se inferir que o TC6 pode subestimar a CF de idosos com DP, devido aos acometimentos motores, como tremor de repouso, rigidez, bradicinesia e instabilidade postural ca- 
racterísticos dessa população, tornando-se uma limitação para a avaliação da CF dessa população.

Os estudos analisados nesta revisão apresentaram limitações importantes, como o reduzido tamanho amostral, a ausência de determinação do estadiamento da DP e do nível de atividade física dos idosos incluídos. Além disso, por se tratar de estudos de corte transversal, não é possível estabelecer uma relação de causa e efeito entre as alterações funcionais observadas nos idosos com DP. Ademais, esta revisão também apresentou algumas limitações que merecem ser destacadas, como a inclusão de apenas cinco estudos, o período definido como critério de busca dos estudos e a exclusão de textos sem disponibilidade livre nos sítios da internet.

Contudo, a presente revisão aponta algumas implicações clínicas relevantes no que tange à necessidade de desenvolvimen- to de novas ferramentas, ou adaptações de testes já validados na literatura para a avaliação da CF de idosos parkinsonianos, instrumentos que levem em consideração os comprometimentos motores inerentes nesses idosos.

\section{Considerações Finais}

Com base nos estudos identificados e na análise realizada nesta revisão, sugere-se que o teste de caminhada de seis minutos não é a ferramenta mais eficaz e fidedigna para a avaliação da capacidade funcional de idosos com doença de Parkinson, pois pode subestimar a sua funcionalidade. Assim, fica evidente a necessidade de novos estudos para o desenvolvimento e a validação de instrumentos de avaliação que contemplem o estadiamento da doença, e sejam capazes de identificar, de forma objetiva, a capacidade funcional nessa população.

\section{REFERÊNCIAS}

1. Haase DCBV, Machado DC, Oliveira JGD. Atuação da fisioterapia no paciente com doença de Parkinson. Fisioter Mov. 2008; 21 (1): 79-85.

2. Rubenis J. A rehabilitational approach to the management of Parkinson's disease. Parkinsonism Relat Disord. 2007; 13 Suppl 3: S495-7.

3. Leddy AL, Crowner BE, Earhart GM. Functional gait assessment and balance evaluation system test: reliability, validity, sensitivity, and specificity for identifying individuals with Parkinson disease who fall. Phys Ther. 2011; 91 (1): 102-13.

4. Jankovic J. Parkinson's disease: clinical features and diagnosis. J Neurol Neurosurg Psychiatry. 2008; 79 (4): 368-76.

5. Gordon PH, Zhao H., Bartley D., et al. Prevalence of Parkinson disease among the Navajo: a preliminary examination. J Parkinsons Dis. 2013; 3 (2): 193-8.

6. Marras C., Tanner CM. Epidemiology of Parkinson's disease. In: Watts RL, Koller WC (Orgs.) Movement disorders: neurologic principles \& practice, 2. ed. New York: McGraw-Hill, 2004, p. 177-95.

7. DATASUS, Fiocruz produzirá medicamentos para tratar mal de Parkinson, 2011. Disponível em: <http://www.blog.saude.gov.br/index.php/voceeosus/29071-3fiocruz-produzira-medicamentopara-tratar-mal-de-parkinson> Acesso em 9 mar. 2017. 
8. Prado RA, Teixeira ALC, Langa CJSO, Egydio PRM, Izzo P. A influência dos exercícios resistidos no equilíbrio, mobilidade funcional e na qualidade de vida de idosas. O Mundo da Saúde. 2010; 34 (2): 183-91.

9. Canning CG, Alison JA, Allen NE, Groeller H. Parkinson's disease: an investigation of exercise capacity, respiratory function, and gait. Arch Phys Med Rehabil, 1997; 78 (2): 199-207. doi: 10.1016/S0003-9993(97)90264-1.

10. Pitts T., Bolser D., Rosenbek J., Troche M., Sapienza C. Voluntary cough production and swallow dysfunction in parkinson's disease dysphagia. Dysphagia, 2008; 23 (3): 297-301.

11. American Thoracic Society (ATS). Committee on Proficiency Standards for Clinical Pulmonary Function Laboratories. ATS statement: guidelines for the six-minute walk test. Am J Respir Crit Care Med., 2002; 166 (1): 111-7. PMid: 12091180.

12. Casas A., Vilaro J., Rabinovich R., et al. Encouraged 6-min walking test indicates maximum sustainable exercise in COPD patients. Chest., 2005; 128 (1): 55-61. doi: 10.1378/chest.128.1.55.

13. Leung ASY, Chan KK, Sykes K., Chan KS. Reliability, validity, and responsiveness of a 2 -min walk test to assess exercise capacity of COPD patients. Chest., 2006; 130 (1): 119-25. doi: 10.1378/ chest.130.1.119.

14. Menezes AM. Metodologia utilizada nos artigos de revisão. J Bras Pneumol., 2011; 37 (4) 1-5.

15. Silva DCL, Vianna E., Martins CP, et al. Perfil dos indivíduos com doença de Parkinson atendidos no setor de fisioterapia de um hospital universitário no Rio de Janeiro. Rev Bras Neurol., 2015; 51 (4): 100-5.

16. Bonjorni LA, Jamami M., Di Lorenzo VAP, Pessoa BV. Influência da doença de Parkinson em capacidade física, função pulmonar e índice de massa magra corporal. Fisioter Mov., 2012; 25 (4): 727-36.

17. Scalzo PL, Flores CR, Marques JR, Robini SCO, Teixeira AL. Impact of changes in balance and walking capacity on the quality of life in patients with Parkinson's disease. Arq Neuropsiquiatr., 2012; 70 (2): 119-24.

18. Falvo MJ, Earhart M. Six-minute walk distance in persons with Parkinson disease: a hierarchical regression model. Arch Phys Med Rehabil., 2009; 90: 1004-7.

19. Canning CG, Ada L., Johnson JJ, McWhirter S. Walking capacity in mild to moderate Parkinson's disease. Arch Phys Med Rehabil., 2006; 87: 371-5.

20. Alves LA, Coelho AC, Brunetto AF. Fisioterapia respiratória na doença de Parkinson idiopática: relato de caso. Fisioter Pesq., 2005; 12 (3): 46-9.

21. Sathyaprabha TN, Kapavarapu PK, Pal PK, Thennarasu K., Raju TR. Pulmonary functions in Parkinson's disease. Indian J Chest Dis Allied Sci., 2005; 47 (4): 251-7. PMid: 16255396.

22. Steffen T., Seney M. Test-retest reliability and minimal detectable change on balance and ambulation tests, the 36-item short-form health survey, and the Unified Parkinson Disease Rating Scale in people with Parkinsonism. Phys Ther 2008; 88: 733-46.

23. Garber CE, Friedman JH. Effects of fatigue on physical activity and function in patients with Parkinson's disease. Neurology 2003; 60: 1119-24.

24. Dibble LE, Hale TF, Marcus RL, Droge J., Gerber JP, LaStayo PC. High-intensity resistance training amplifies muscle hypertrophy and functional gains in persons with Parkinson's disease. Mov Disord 2006; 21: 1444-52.

25. Ferreira LL, Cochito TC, Caíres F., Marcondes LP, Saad PCB. Functional capacity of institucionalized elderly with and without Alzheimer's disease. Rev Bras Geriatr Gerontol. 2014; 17 (3): 567-73. 


\section{DADOS DOS AUTORES}

\section{LUCAS LIMA FERREIRA}

Mestre em Fisioterapia pela Universidade Estadual Paulista Júlio de Mesquita Filho. Docente da PósGraduação em Fisioterapia Cardiorrespiratória na Faculdade Medicina São José do Rio Preto. São José do Rio Preto/SP - Brasil. lucas_lim21@hotmail.com

\section{ODETE MAUAD CAVENAGHI}

Mestre em Ciências da Saúde pela Faculdade de Medicina de São José de Rio Preto. Docente da PósGraduação em Fisioterapia Cardiorrespiratória na Faculdade Medicina São José do Rio Preto. São José do Rio Preto/SP - Brasil. bebelmauad2009@hotmail.com

Submetido em: 18-5-2017

Aceito em: 15-7-2017 